OPEN ACCESS

Edited by:

Nora S. Vyas,

Kingston University,

United Kingdom

Reviewed by:

Kirby Deater-Deckard,

University of Massachusetts Amherst,

United States

Rajiv Radhakrishnan,

Yale University, United States

${ }^{*}$ Correspondence:

Akemi Tomoda

atomoda@u-fukui.ac.jp

Specialty section:

This article was submitted to

Child and Adolescent Psychiatry,

a section of the journal

Frontiers in Psychiatry

Received: 12 June 2017

Accepted: 30 January 2018

Published: 14 February 2018

Citation:

Nishikawa S, Fujisawa TX, Kojima M and Tomoda A (2018) Type and

Timing of Negative Life

Events Are Associated with

Adolescent Depression.

Front. Psychiatry 9:41.

doi: 10.3389/fpsyt.2018.00041

\section{Type and Timing of Negative Life Events Are Associated with Adolescent Depression}

\author{
Saori Nishikawa, Takashi X. Fujisawa, Masahiko Kojima and Akemi Tomoda* \\ Research Center for Child Mental Development, Fukui University, Fukui, Japan
}

Previous studies have demonstrated an association between negative life events (NLEs) in childhood and resilience/posttraumatic growth (PTG) with regard to the pathogenesis of major depressive disorder. We hypothesized that the type and timing of NLEs interact to influence mental health in the general youth population. Therefore, the present study aimed to examine the effects of NLE timing and intensity on current depressive symptoms, and to determine the direct and indirect effects of NLEs/resilience on PTG and depression among non-clinical adolescents. Data were collected from 1,038 high-school students across seven high schools in Fukui, Japan, during their freshman and sophomore years (648 boys and 390 girls, mean age $=15.71$, SD $=0.524$ ). Respondents completed a set of questionnaires designed to evaluate the type and timing of NLEs, depressive and traumatic symptoms, and PTG. Cluster analysis was used to divide participants into three groups based on outcomes: "cluster 1 " $(n=631)$, for whom depressive scores were significantly lower than other two subgroups ( $p<0.05$, for both); "cluster 2" ( $n=52$ ), for whom levels of current and past perceived stress associated with NLEs were significantly higher than those of the other two subgroups $(p<0.05$, for both); "cluster 3 " $(n=374)$, for whom perceived stress at the time of NLE was significantly higher than that of participants in the cluster $1(p<0.05)$ group, but not the cluster 2 group. Our findings indicated that exposure to NLEs at a younger age resulted in stronger negative outcomes and that NLE timing and intensity were associated with PTG and current symptoms of depression. Furthermore, path analysis demonstrated that associations between perceived stress at the time of NLEs were direct and indirect predictors of current depression via PTG and that posttraumatic stress symptom and PTG mediate the association between NLEs/trait-resiliency and current depression. In conclusion, our findings suggest that event intensity, NLE timing, and gender may play a role in emotional vulnerability during adolescence.

Keywords: negative life event, trait-resilience, posttraumatic stress symptoms, posttraumatic growth, adolescence

\section{INTRODUCTION}

Most individuals have been exposed to some level of trauma during childhood. Previous research has indicated that negative life events (NLEs) such as bullying, traffic accidents, natural disasters, interpersonal problems, conflicts with parents, and poor academic performance are among the most robust predictors of poor well-being in adolescence (1-4). Additional research has suggested that 
middle school students experience and respond to NLEs differently than older students and that such differences contribute to heightened emotional vulnerability and more frequent emotional reactions (5).

Several studies have also reported that NLEs during childhood are significant predictors of psychiatric disorders in adulthood (6, 7 ) and that specific NLEs are associated with emotional problems $(6,8)$. For example, health-threatening events and stressful interpersonal relationships have been linked to depressive symptoms (6). Moreover, adolescents tend to be more vulnerable to depression, anger, and anxiety associated with NLEs (5). Exposure to traumatic events has also been identified as a risk factor for posttraumatic stress disorder (PTSD) and anxiety (9). Complex trauma is regarded as both exposure to multiple and/or chronic interpersonal traumatic experiences typically occurring within the caregiving system and the immediate and ongoing impact of this exposure across areas of development and functioning (10). Studies have revealed that individuals exposed to trauma during middle childhood report higher levels of depressive and PTSD symptoms in adulthood, relative to non-exposed individuals (11). Teicher and Samson (12) proposed the term "ecophenotype" to distinguish psychiatric conditions (e.g., major depressive disorder, anxiety, PTSD) based on the timing, type, and severity of exposure to maltreatment. Accumulating evidence suggests that emotional dysregulation plays a mediating role in the association between exposure to violence and posttraumatic stress symptoms (PTSS) in clinically referred adolescents (13).

However, not all adolescents exposed to NLEs experience poor mental health outcomes. In fact, previous studies have suggested that some individuals experience positive outcomes (14, 15) following NLE (e.g., breaking away from negative influences, triumphing over adversity, etc.). Tedeschi and Calhoun (15) defined posttraumatic growth (PTG) as "a phenomenon of a personal development as positive change in a person's life as a result of highly traumatic events." Early research on the relationship between NLEs and PTG demonstrated an association between fearfulness relating to the degree of stress and high levels of PTG (16). A more recent meta-analysis reported that this relationship approximates a "U-shaped" curve, and that PTG is similarly correlated with PTSS (17). That is, the association between PTG and PTSS intensity is such that very high or very low levels of stress are more likely to result in PTG (18). Additional studies have indicated that PTG leads to the development of resilience, indirectly inhibiting depressive symptoms and PTSS associated with additional NLEs $(19,20)$. Other studies have reported that PTG reduces later symptoms stress (21) but not those associated with depression (22).

Trait-resilience (ego-resiliency) is defined as the ability to adjust to environmental stress and change $(23,24)$. Trait-resilience is stable across childhood (25) and into adulthood (26), and previous studies have indicated that interactions between genes and environment play a role in regulating trait resilience. Taylor et al. (27) demonstrated that parenting style and genetic variations in serotonin levels are significant predictors of trait-resilience during early childhood. Individuals with high trait-resilience exhibit higher levels of adaptive flexibility during recovery from traumatic experiences (25). In addition, trait-resilience is positively associated with virtues (i.e., conscientiousness, vitality, and relationship) and PTG, while PTSD symptoms are not (28). One clinical study demonstrated that, while neuroticism and extraversion mediate the association between positive life events and resilience, NLEs directly contribute to trait-resilience (29). Resilience has also been associated with NLEs and mental health problems (30), acting as a mediating factor in the association between childhood maltreatment and psychiatric symptoms later in life (31).

Despite such findings, little is known regarding the risk and protective factors that characterize resilience and PTG among Japanese adolescents. Early research regarding PTSS focused on negative/stressful life events that are likely to cause narrowly defined trauma (e.g., wars and disasters), while few studies have focused on PTSS associated with NLEs in daily life. Thus, it is necessary to elucidate the effects of PTG and resilience on PTSS associated with NLEs. The present study aimed to examine the effects of NLE timing and intensity on current depressive symptoms and to determine the direct and indirect effects of NLEs/resilience on PTG and depression among non-clinical adolescents in Japan. We hypothesized (a) that NLE timing and stress intensity are associated with the development of PTG and current depressive symptoms, (b) that NLEs exert both direct and indirect effects on PTG and depression (see Figures 1A,B), and (c) that PTSS and PTG mediate the association between stress and current depressive outcomes (e.g., depression, stress related to NLEs, see Figure 1B).

\section{MATERIALS AND METHODS}

\section{Participants}

The present study included 1,055 high-school students from Fukui prefecture (778,550 inhabitants). As 17 students were

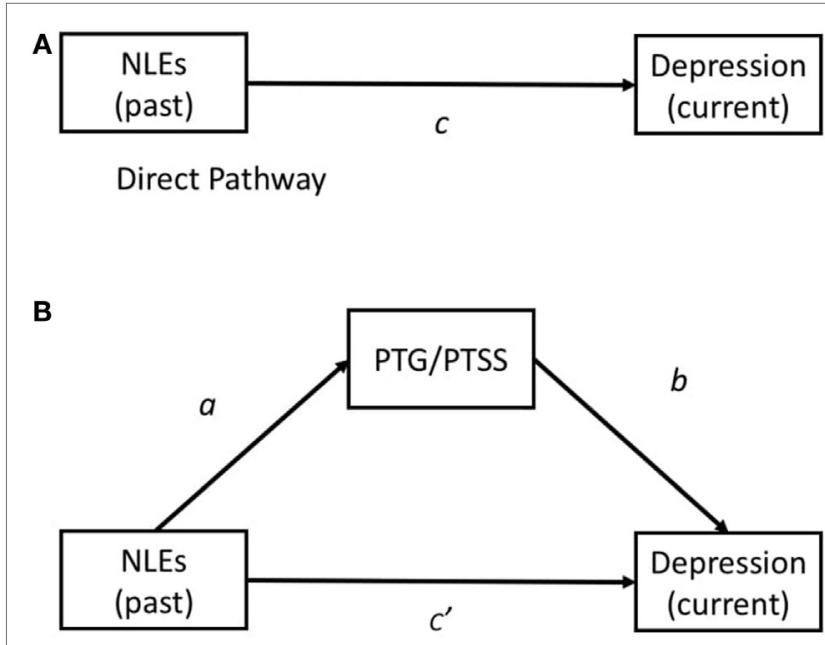

Indirect or Mediated Model

FIGURE 1 | (A) Illustration of the direct effect of NLEs (past stress) on current depressive symptoms. (B) Illustration of a mediation model in which NLEs (past) are hypothesized to exert direct and indirect effects on depression via PTG or PTSS. Abbreviations: NLE, negative life event; PTG, posttraumatic growth; PTSS, posttraumatic stress symptoms. 
absent at the time of the study, a final total of 1,038 students (mean age $=15.71, \mathrm{SD}=0.524$, range $=15-17$ years; boys $=648$, girls $=390)$ were included $($ see Table $\mathbf{1})$.

\section{Procedure}

The study protocol was approved by the ethical committee at the University of Fukui (approval number: 834). The purposes of the present study were announced at a meeting of high-school principals from Fukui Prefecture, at which time their assistance and cooperation were requested. Principals and individual classroom teachers provided consent to include 29 classes from seven high schools. A researcher and technician visited schools and conducted the study. The purposes and voluntary nature of the present study were explained to parents by a letter and to students orally and in writing, following which they were requested to submit a letter of consent if they wished to participate in the study. Participating students completed the questionnaires anonymously during regular class hours. Identifying information included only gender, age and class. Students took approximately $30 \mathrm{~min}$ to complete the questionnaires.

\section{Measures}

\section{Type and Timing of NLEs}

Participants were asked to select the types of stressful or traumatic life events they had experienced from among a list of the following 10 options: death of family member/close friend, parental divorce, conflict with parents, academic failure, domestic economic issues, bullying, interpersonal conflicts, serious illness or injury, accident or disaster, and other. Participants were then asked to rate how much stress they had experienced at the time of the NLE, as well as their current level of stress associated with the NLE, on a Likert scale ranging from 1 (low stress) to 4 (high stress).

\section{Posttraumatic Stress Symptom}

Following the NLE questionnaire, participants completed the Impact of Event Scale-Revised (IES-R), which includes 22 items across three subscales designed to assess symptoms of posttraumatic stress: intrusion, avoidance, and hyperarousal in the past 7 days (32). Each item was rated on a five-point Likert scale, ranging from 0 (not at all) to 4 (a very great degree). Higher scores indicate higher levels of traumatic symptoms. The Japanese version of the IES-R has demonstrated good validity and test-retest reliability in previous studies (33).

\section{Posttraumatic Growth}

The Japanese version of the Posttraumatic Growth Inventory (PTGI-J) (34) was developed based on the original version of the

TABLE 1 | Demographic characteristics (age and gender) of the participants.

\begin{tabular}{lcccccc}
\hline & \multicolumn{9}{c}{ Age } & & $\boldsymbol{t}$ & $\boldsymbol{p}$-Value \\
\cline { 2 - 5 } & Mean & SD & Minimum & Maximum & & \\
\hline Boys $(n=648)$ & 15.68 & 0.51 & 15 & 18 & 2.50 & 0.013 \\
Girls $(n=390)$ & 15.77 & 0.55 & 15 & 18 & & \\
Total $(n=1,038)$ & 15.71 & 0.52 & 15 & 18 & - & -
\end{tabular}

PTGI (35), which consists of 21 items designed to assess levels of PTG in individuals who have experienced traumatic events. Participants were asked to provide responses on a 6-point Likert scale ranging from 0 ( $I$ did not experience this change as a result of my crisis $)$ to $5=(I$ experienced this change to a very great degree as a result of my crisis).

\section{Depression}

The severity of depressive symptoms was evaluated using the Birleson Depression Self-Rating Scale for Children (DSRS-C) (36), which consists of 18 items designed to assess symptoms of depression within the past week. Responses were provided on a Likert scale ranging from 0 (never) to 2 (mostly). The validity and reliability of the Japanese version of the DSRS- $C$ have been confirmed in a previous study (37).

\section{Trait Resilience}

The Ego-Resiliency Scale (ER-89) (38) is a unidimensional, 14-item self-report scale designed to measure trait resilience (i.e., ego-resiliency) in adolescents. Participants provided responses on a 4-point Likert scale, ranging from 1 (does not apply at all) to 4 (applies very strongly). The Japanese version of the ER-89 has demonstrated good reliability and validity in previous studies (39).

\section{Variables and Statistical Methods}

The Statistical Package for Social Sciences (SPSS) version 22 (IBM, 2013) was used for performing descriptive statistics, correlations, and analyses of variance (ANOVAs). Path models developed using Structural Equation Models on AMOS 22 were used to evaluate the relationships among variables. To investigate whether a variable (PTG) is a mediator between independent variable (NLEs past) and dependent variable (depression current) in the path analysis, we first drew a direct path from NLEs past to depression current (Figure 1A). In the next analysis, two paths were added (Figure 1B): one from NLEs past to PTG and another from $P T G$ to depression current. If $P T G$ is a significant mediator, the weight of the path from NLEs past to depression current will decrease in the second analysis, relative to that in the first analysis (40). The goodness of fit index (GFI) is considered a reasonable statistical index for evaluating a model and was used to assess the fit between a hypothesized model and the data. AMOS was used to evaluate the following parameters of each model based on different theoretical perspectives: CMIN/df (the minimum value of sample discrepancy divided by its degree of freedom), in which smaller values are preferable (41), the comparative fit index (CFI), a measure of the relative amount of variance and covariance (values over 0.9 are preferable) (42), the root mean square error of approximation (RMSEA) based on population discrepancy (values below 0.08 are preferable) (43), and the incremental fit index (IFI) (values over 0.9 are preferable) (44).

\section{RESULTS}

\section{Descriptive Statistics}

The distributions of variables (ER-89, PTSS, depression) were approximately bell-shaped, with the exception of those for past 
NLEs (stress intensity at the time of NLE), current NLEs (current stress intensity associated with the NLE), and PTSS (which exhibited peaks at the low end). Table 2 shows the means (SDs, skewness, kurtosis) and correlations between PTSS, DSRS-C scores, ER-89 scores, and other variables. PTSS was positively correlated with DSRS-C scores, PTG, past NLEs, current NLEs ( $r=$ between -0.17 and $-0.23, p<0.001$ and 0.05 ), and negatively correlated with ER-89 scores $(r=-0.10, p=0.001)$. PTG was positively correlated with ER-89 scores and past NLEs ( $r=$ between -0.17 and $-0.23, p<0.001$ and 0.05$)$, and negatively correlated with DSRS-C scores and current NLEs ( $r=$ between -0.17 and -0.23 , $p<0.001$ and 0.05$)$. Gender $(1=$ boy, $2=$ girl $)$ was positively correlated with PTSS, DSRS-C scores, past NLEs, and current NLEs. Further correlational analysis was conducted separately based on gender. Trait-resilience and PTSS were negatively correlated among girls only $(r=-0.15, p=0.004)$, while PTG and PTSS were positively correlated among boys only $(r=0.17, p=0.000)$. One-way ANOVA indicated that girls scored significantly higher on the DSRS-C $(p=0.01)$ and had significantly higher levels of PTSS $(p=0.000)$, stress intensity at the time of NLEs $(p=0.000)$, and current stress associated with NLEs $(p=0.021)$ than boys.

Table 3 lists the frequencies and percentages of NLEs, the timing of exposure (age group), and the mean values of the other variables. A univariate $F$-test revealed a significant main effect of NLE timing on depressive symptoms, PTSS, and PTG. Post hoc analysis indicated that individuals who experienced NLEs after high school (high-school group) reported significantly higher levels of depressive symptoms than those who experienced NLEs in junior high school $(p<0.05)$. The high-school group also exhibited significantly higher levels of PTSS than elementary school and junior high-school groups $(p<0.05$ for both), indicating that more recent NLEs had a greater impact on outcomes. Furthermore, a univariate F-test excluding participants ( $n=255$ ) who reported more recent NTEs (high-school group) revealed a significant main effect of NLE timing on PTSS ( $p<0.05$ for both). Post hoc analysis indicated that individuals who experienced NLEs before elementary school ( $\leqq 6$ years old) reported significantly higher levels of PTSS than those who had experienced such events in elementary or junior high school $(p<0.05$ for both).

\section{Type and Timing of NLEs}

We used SPSS 22 to perform a cluster analysis of the sample of 1,038 adolescents in order to identify the subtypes of mental health variables (i.e., depression, PTSS, PTG, perceived past/current stress) in relation to NLE types. First, mean values of mental health variables were calculated for each NLE types. Clustering was performed using Ward's linkage and agglomeration schedule. Three cluster solutions were identified into the following subgroups: cluster 1, cluster 2, and cluster 3. Participants in the first cluster $(n=631)$ reported significantly lower levels of depression than those in the second and third clusters ( $p<0.05$, for both). Participants of the second cluster $(n=52)$ reported significantly higher current and past NLEs associated with NLEs than those of the cluster 1 and cluster 2 ( $p<0.05$ for both). Participants of the third cluster $(n=374)$ reported experiencing more stress at the time of the NLE than those of the first cluster $(p<0.05)$, but not those of the second cluster. Finally, participants of both the cluster 1 and cluster 3 groups reported higher levels of PTG than those of the cluster 2 ( $p<0.05$, for both). There were no significant differences in trait-resilience scores among the three groups (Figure 2). Table 4 shows the cluster characteristics identified

TABLE 2 | Means, SDs, and correlations among study variables.

\begin{tabular}{|c|c|c|c|c|c|c|c|c|c|c|}
\hline & Mean (SD) & Skewness & Kurtosis & 1 & 2 & 3 & 4 & 5 & 6 & 7 \\
\hline 1. Gender ( 1 = boys, 2 = girls) & $1.38(0.49)$ & 0.514 & -1.739 & - & & & & & & \\
\hline 2. Posttraumatic stress syndrome & $21.15(17.48)$ & 0.748 & -0.098 & $0.141^{\star \star}$ & - & & & & & \\
\hline 3. Depressive symptoms & $11.61(5.87)$ & 0.443 & -0.022 & $0.078^{\star}$ & $0.440^{\star \star}$ & - & & & & \\
\hline 4. Trait resilience & $21.18(7.84)$ & 0.049 & -0.317 & -0.041 & $-0.099^{\star \star}$ & $-0.572^{\star \star}$ & - & & & \\
\hline 5. PTG & $44.71(22.24)$ & -0.086 & -0.670 & 0.016 & $0.136^{\star \star}$ & $-0.376^{\star \star}$ & $0.516^{\star \star}$ & - & & \\
\hline 6. Past NLEs (stress) & $2.82(0.98)$ & -0.295 & -0.987 & $0.177^{\star \star}$ & $0.360^{\star \star}$ & $0.226^{\star \star}$ & -0.001 & $0.136^{\star \star}$ & - & \\
\hline 7. Current NLEs (stress) & $1.68(0.82)$ & 1.146 & 0.761 & $0.071^{\star}$ & $0.375^{\star \star}$ & $0.391^{\star \star}$ & $-0.162^{\star \star}$ & $-108^{\star \star}$ & $0.368^{\star \star}$ & - \\
\hline
\end{tabular}

${ }^{*} p<0.05,{ }^{* *} p<0.001$. NLES, negative life events; PTSS, posttraumatic stress symptoms; PTG, posttraumatic growth; Past NLES, stress intensity at the time of NLE, Current NLES, current stress intensity associated with the NLE.

TABLE 3 | Mean scores (SDs) of adolescents in each age group based on the NLE timing.

\begin{tabular}{|c|c|c|c|c|c|c|}
\hline & \multicolumn{3}{|c|}{ Age at time of NLEs } & \multirow[t]{2}{*}{$\boldsymbol{F}$} & \multirow[t]{2}{*}{$p$-Value } & \multirow[t]{2}{*}{ Post hoc comparisons } \\
\hline & A. before elementary & B. elementary school & C. junior high school & & & \\
\hline Depression & $11.92(6.47)$ & 11.70 (6.02) & $11.08(5.72)$ & 1.04 & ns & ns \\
\hline Trait-resilience & $19.43(8.12)$ & 20.50 (8.13) & $21.69(7.52)$ & 2.85 & ns & ns \\
\hline PTSS & $27.32(23.51)$ & $19.60(17.00)$ & $21.07(17.13)$ & 3.20 & 0.027 & $A>B$ and $C$ \\
\hline PTG & 38.32 (20.72) & 42.97 (1.62) & 46.35 (21.87) & 3.63 & 0.041 & $A<B$ and $C$ \\
\hline Past NLEs & $2.62(1.26)$ & $2.73(1.02)$ & $2.83(0.94)$ & 1.26 & ns & $\mathrm{ns}$ \\
\hline Current NLEs & $1.89(1.10)$ & $1.46(0.71)$ & $1.60(0.76)$ & 5.39 & 0.005 & $A>B$ and $C$ \\
\hline
\end{tabular}

NLES, negative life events; PTSS, posttraumatic stress symptoms; PTG, posttraumatic growth; Past NLES, stress intensity at the time of NLE; Current NLES, current stress intensity associated with the NLE. 
by the pairwise comparisons, while Table 5 shows the results of post hoc analysis.

\section{Path Analysis}

After the correlational analysis, the following strategy was used to examine the direct and indirect effects of each variable. The estimated model with standardized path coefficients is presented in Table 6. First, a direct-pathway model was constructed using past NLEs (stress at the time of NLEs) as a predictor of current depression. The pathway linking past NLEs and depression was significant $(p<0.001$, see Figure 3A), with acceptable GFIs $[F(1,9)=3.90, p=0.048 ; \mathrm{CMIN} / \mathrm{df}=3.895, \mathrm{CFI}=0.946$, RMSEA $=0.530$, IFI $=0.946]$.

Model 1 was developed in order to assess the direct and indirect effects of PTG as a mediator of depression (see Figure 3B). The pathways linking NLEs, PTG, and depression were all significant $(p<0.001$ for all), indicating a partial mediation. All exhibited acceptable GFIs $[F(1,9)=9.018, p=0.003$; CMIN/ $\mathrm{df}=9.018, \mathrm{CFI}=0.971$, RMSEA $=0.088$, IFI $=0.971$, see Table 6]. All paths in Model 1 were significant across gender $(p<0.001)$. However, GFIs appeared to be non-acceptable for boys $[F(1,9)=9.634, p=0.002 ; \mathrm{CMIN} / \mathrm{df}=9.634, \mathrm{CFI}=0.950$, RMSEA $=0.116$, IFI $=0.951]$, while a good fit was observed among girls $[F(1,9)=0.538, p=0.463 ; \mathrm{CMIN} / \mathrm{df}=0.538$, CFI $=1.000$, RMSEA $=0.000$, IFI $=1.004$; see Table 6]. Model 2 was constructed to assess the direct and indirect effects of PTSS as a mediator of depression (see Figure 3C). The pathways linking NLEs, PTSS, and depression were all significant $(p<0.001$ for all), and all exhibited acceptable GFIs $[F(1,9)=8.80, p=0.003$; $\mathrm{CMIN} / \mathrm{df}=8.803, \mathrm{CFI}=0.929, \mathrm{RMSEA}=0.087, \mathrm{IFI}=0.979$; see Table 6]. Good GFIs were observed among girls $[F(1,9)=0.04$, $p=0.837 ; \mathrm{CMIN} / \mathrm{df}=0.042, \mathrm{CFI}=1.000, \mathrm{RMSEA}=0.000$, IFI $=1.005]$, while the model did not fit the data very well among boys (see Table 6).

Model 3 (See Figure 4) was constructed with multiple mediators (i.e., PTSS, PTG), in which NLEs exhibited direct and indirect effects on depression (current) via PTSS and PTG. The pathways linking NLEs, PTG, PTSS, and depression were all significant $(p<0.001$ for all), and all exhibited acceptable GFIs $[F(1,14)=9.08, p=0.003 ; \mathrm{CMIN} / \mathrm{df}=9.076, \mathrm{CFI}=0.988$, RMSEA $=0.088$, IFI $=0.988$, see Table 6].

Finally, Model 4 (Figure 5) was constructed using predictors (NLEs past and trait-resilience) as well as outcome variables (depression and NLEs current) in order to assess the direct and indirect effects of any mediating variables (PTSS and PTG). The pathways linking NLEs past, PTG, trait-resilience, and depression

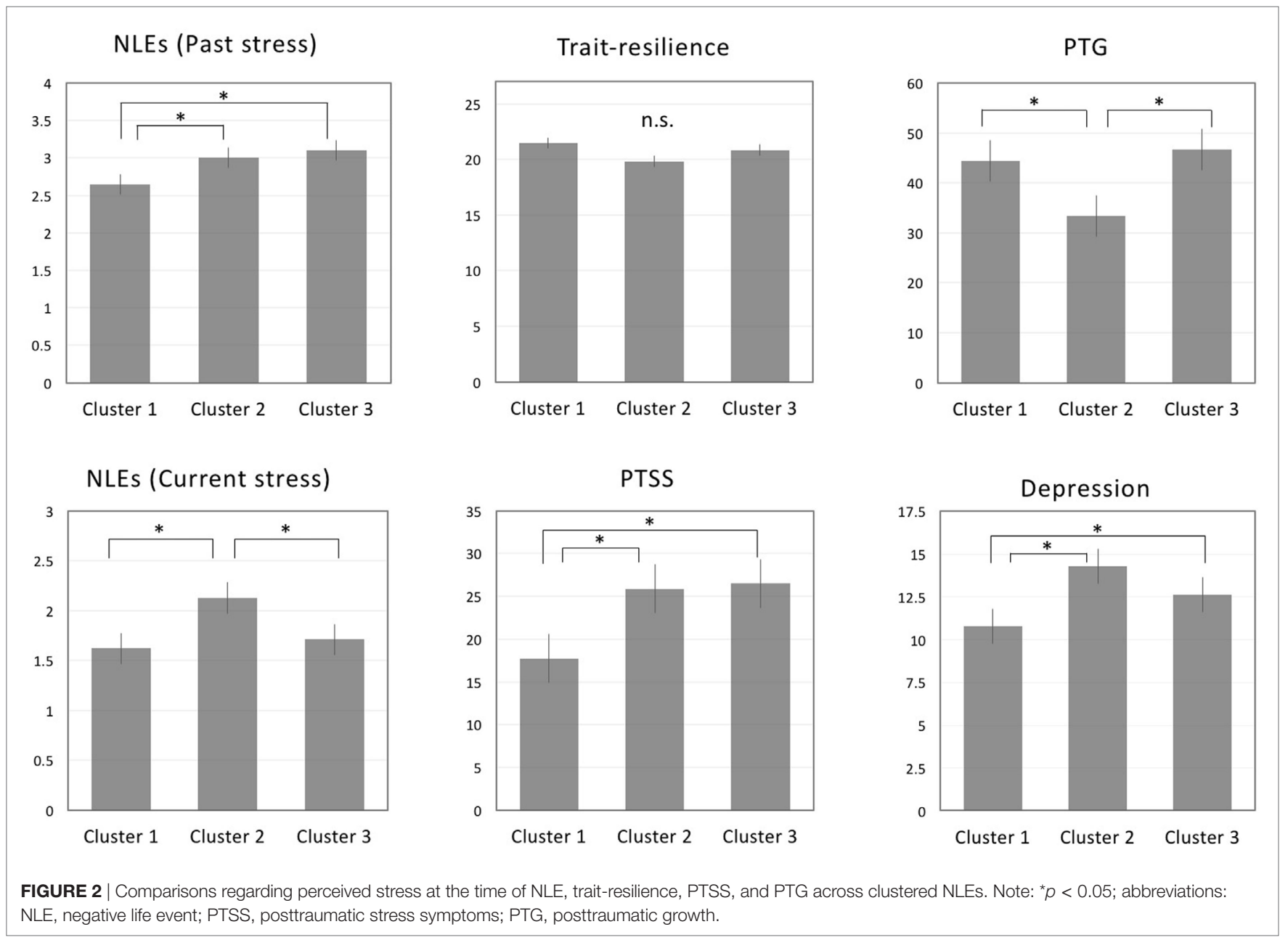




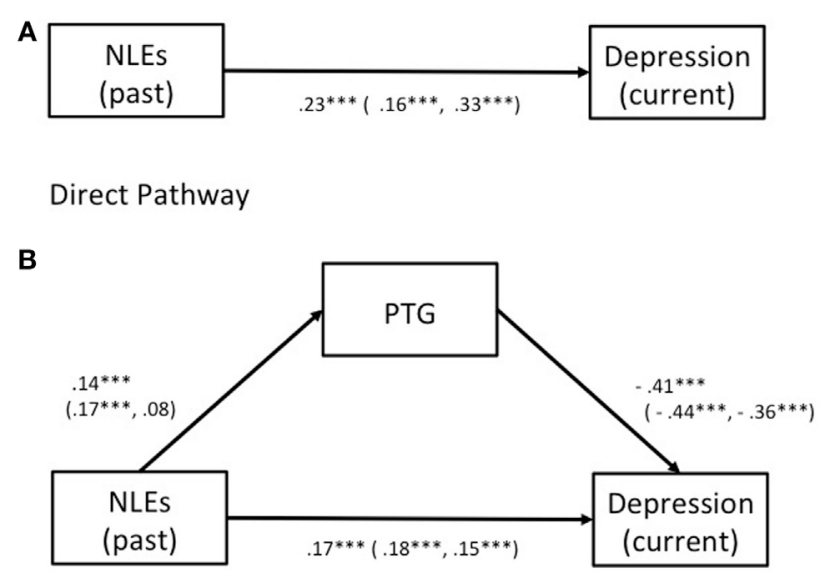

Result of Indirect or Mediated Model (Model 1)

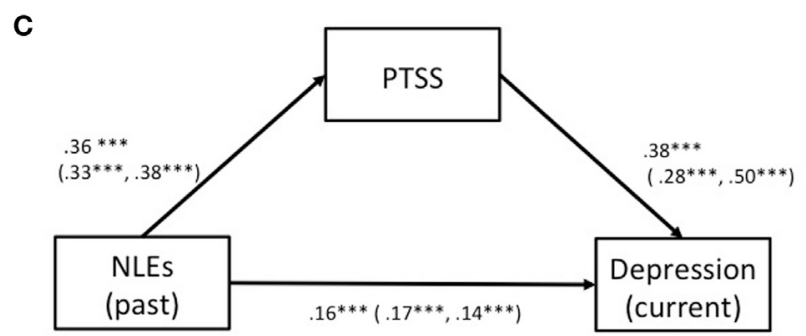

Result of Indirect or Mediated Model (Model 2)

FIGURE 3 | (A) Results of direct model in which negative life events (NLEs) (past stress) as a predictor of current depression. (B) Results of a mediation model in which NLEs (past stress) are hypothesized to exert direct and indirect effects on depression via posttraumatic growth (PTG). (C) Results of a mediation model in which NLEs (past stress) are hypothesized to exert direct and indirect effects on depression via PTSS. Note: standardized estimates of the direct and indirect effects on the depressive symptoms presented for all participants (male, female), respectively.

were all significant ( $p<0.001$ and $p<0.005$, see Figure 4), thus suggesting a partial mediation. The overall model fit the data well, $F(2,27)=2.388, p=0.303 ; \mathrm{CMIN} / \mathrm{df}=1.19$, CFI $=1.000$, RMSEA $=0.000$, IFI $=1.000$ (see Table 6), indicating that PTSS and PTG mediate the effects of past NLEs and trait-resilience on current depressive symptoms and stress associated with NLEs. Additionally, we used a bootstrap examination method (with 5,000 bootstrap samples). If the CIs did not include 0 , it could indicate that the mediating effects were statistically significant (45). In the present study, the mediating effects (expect a path model linking between trait-resilience and depression) were all significant. The results of bootstrap analysis indicated that the indirect effect of past stress on depression via PTG or PTSS were significantly different from 0 (see Data Sheet S1 in Supplementary Material).
TABLE 4 | Cluster groupings based on NLE type.

\begin{tabular}{lll}
\hline Types of negative life event & \% $(\boldsymbol{n})$ & Cluster \\
\hline 1. Death of family member/close friend & $11.5(119)$ & Cluster 1 \\
2. Parental divorce & $3.5(36)$ & Cluster 3 \\
3. Conflict with parents & $5.0(52)$ & Cluster 2 \\
4. Academic failure & $27.6(286)$ & Cluster 1 \\
5. Domestic economic issues & $6.6(69)$ & Cluster 1 \\
6. Bullying & $23.0(239)$ & Cluster 3 \\
7. Interpersonal conflicts & $9.5(99)$ & Cluster 3 \\
8. Serious illness/injury & $1.1(11)$ & Cluster 1 \\
9. Accident or disaster & $12.2(127)$ & Cluster 1 \\
\hline
\end{tabular}

The study conducted the analysis separately in gender. Except for the path linking past NLEs and PTG among girls, all paths in Model 3 were significant across gender ( $p<0.001$, see Figure 4). Among both boys and girls, all paths in Model 4 (see Figure 5) were significant $(p<0.001$ and $p<0.05$, see Table 6). GFIs appeared acceptable for boys $[F(2,27)=1.466, p=0.481, \mathrm{CMIN} /$ $\mathrm{df}=0.733, \mathrm{CFI}=1.000, \mathrm{RMSEA}=0.000, \mathrm{IFI}=1.001]$ and girls $[F(2,27)=3.728, p=0.155 ; \mathrm{CMIN} / \mathrm{df}=1.864, \mathrm{CFI}=0.997$, RMSEA $=0.047$, IFI $=0.999$; see Figure 5].

\section{DISCUSSION}

The primary aims of the present study were to examine the effects NLE timing and intensity on PTSS, PTG, and depressive symptoms; and to explore the direct and indirect effects of NLEs on PTG and depression among non-clinical adolescents in Japan. Our findings indicated that NLE timing and intensity were associated with PTG and current symptoms of depression. Furthermore, path analysis demonstrated that associations between perceived stress at the time of NLEs were direct and indirect predictors of current depression via PTG and that PTSS and PTG mediate the association between NLEs/trait-resiliency and current depression.

Although trait-resilience was not associated with perceived stress concerning a particular NLE in the present study, our findings indicate that this factor directly and indirectly influences current depression among adolescents. The present results are consistent with those of previous studies $(30,31)$, which have demonstrated associations among resilience, child maltreatment, and later depressive symptoms. Thus, traitresilience may be regarded as an independent construct that relies on the level of PTG to indirectly improve symptoms of depression.

In accordance with the findings of early studies $(6,8)$, our findings indicated that different types of NLEs were associated with variations in PTG levels and outcomes (e.g., PTSS and depression).

Among the three cluster-groups of the present study, adolescents who reported conflict with parents (i.e., cluster 2 ) tended to have higher levels of PTSS and lower levels of PTG, while those of the cluster 1 or cluster 3 exhibited higher levels of PTG, resulting in lower levels of PTSS and current depression. Previous studies have indicated that relationships with parents influence well-being during childhood and adolescence (46). 
TABLE 5 | Mean scores (SDs) of adolescents classified by cluster group.

\begin{tabular}{|c|c|c|c|c|c|c|}
\hline & \multicolumn{3}{|c|}{ Group } & \multirow[t]{2}{*}{$\boldsymbol{F}$} & \multirow[t]{2}{*}{$p$-Value } & \multirow[t]{2}{*}{ Post hoc comparisons } \\
\hline & Cluster 1 & Cluster 2 & Cluster 3 & & & \\
\hline Depression & $10.79(5.62)$ & $14.31(5.71)$ & $12.65(6.05)$ & 17.74 & 0.000 & Cluster $2>$ Cluster 1 , Cluster 3 \\
\hline Trait-resilience & $21.48(7.90)$ & $19.83(8.02)$ & $20.85(7.68)$ & 1.55 & ns & \\
\hline PTSS & $17.75(16.07)$ & $25.90(18.29)$ & 26.48 (18.29) & 8.45 & 0.000 & Cluster 2 > Cluster 1 \\
\hline PTG & $44.50(22.32)$ & 33.35 (21.79) & $46.75(21.71)$ & 32.14 & 0.000 & Cluster 1 , Cluster $2>$ Cluster 3 \\
\hline Past NLEs & $2.65(0.99)$ & $3.00(0.97)$ & $3.10(0.88)$ & 26.06 & 0.000 & Cluster $2>$ Cluster 1 , Cluster 3 \\
\hline Current NLEs & $1.62(0.79)$ & $2.13(1.10)$ & $1.71(0.82)$ & 9.88 & 0.000 & Cluster $2>$ Cluster 1 , Cluster 3 \\
\hline
\end{tabular}

NLES, negative life events; PTSS, posttraumatic stress symptoms; PTG, posttraumatic growth; Past NLEs, stress intensity at the time of NLE; Current NLES, current stress intensity associated with the NLE.

TABLE 6 | Fit indices of the models.

\begin{tabular}{lccccc}
\hline Model & $\boldsymbol{x}^{\mathbf{2}}(\mathbf{d f})$ & CMIN/df & CFI & RMSEA & IFI \\
\hline Model 1 (All) & $9.02(1)$ & 9.018 & 0.971 & 0.088 & 0.971 \\
Boys & $9.63(1)$ & 9.63 & 0.950 & 0.116 & 0.951 \\
Girls & $0.54(1)$ & 0.538 & 1.00 & 0.000 & 1.000 \\
Model 2 (All) & $8.80(1)$ & 8.803 & 0.979 & 0.087 & 0.979 \\
Boys & $10.61(1)$ & 10.609 & 0.936 & 0.122 & 0.938 \\
Girls & $0.04(1)$ & 0.042 & 1.000 & 0.000 & 1.005 \\
Model 3 (All) & $9.08(1)$ & 9.076 & 0.988 & 0.088 & 0.988 \\
Boys & $9.61(1)$ & 9.605 & 0.977 & 0.115 & 0.977 \\
Girls & $1.51(1)$ & 1.512 & 0.998 & 0.036 & 0.998 \\
Model 4 (All) & $2.34(2)$ & 2.388 & 1.00 & 0.014 & 1.000 \\
Boys & $1.47(2)$ & 0.733 & 1.000 & 0.000 & 1.001 \\
Girls & $3.73(2)$ & 1.864 & 0.997 & 0.047 & 0.998 \\
\hline
\end{tabular}

Note, CMIN/df, the minimum value of sample discrepancy divided by its degree of freedom.

CFI, comparative fit index; RMSEA, root mean square error of approximation; IFI, incremental fit index.

As adolescence is the period during which individuals begin to develop autonomy and independence (47), parent-child conflict is more likely to occur. However, in the present study, conflict with parents was the most robust predictor of poor outcomes such as current depression, high PTSS, and low PTG. These findings are consistent with those of a previous study, which demonstrated that parenting style had a direct effect on PTSS via resilience (48). We observed that depressive symptoms were less severe in students who had experienced trauma from cluster 1 , indicating that some students may have been able to overcome such life events (e.g., death of family member, academic failure, etc.). Furthermore, the cluster 1 trauma group exhibited less severe depression than the cluster 2 group, in which conflicts with parents were noted, indicating that such conflicts may continue to affect the mental health of students. However, we did not investigate the types of parental conflicts with which students were struggling. As the severity of these conflicts may also differ among students, further studies are required to examine the influence of various types of parental conflict.

The results of the present study are consistent with those of Mann et al. (5), who reported that adolescents are more vulnerable than young adults to more recent NLEs even when they are relatively low in intensity. Our findings suggest that exposure to NLEs at a younger age resulted in stronger negative outcomes during adolescence, in accordance with the findings

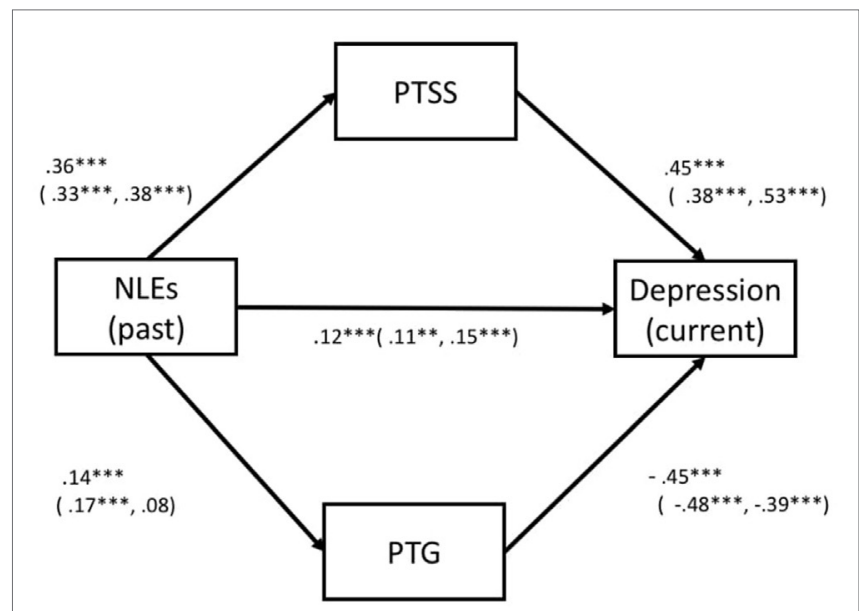

FIGURE 4 | Multi and mediated model (Model 3). Illustration of a mediation model in which NLEs (past) is hypothesized to exert indirect effect on current depression associated with NLEs (past) via PTSS and PTG. Note: standardized estimates of the direct and indirect effects on depressive symptoms are presented for all participants (male, female), respectively. Abbreviations: NLE, negative life event; PTSS, posttraumatic stress symptoms; PTG, posttraumatic growth.

of Dunn et al. (11). Adolescents exposed to NLEs prior to reaching school age reported higher levels of PTSS and PTG than those who experienced NLEs in elementary and junior high school. These results suggest that individuals exposed to NLEs prior to the age of 6 are more emotionally vulnerable than non-exposed individuals during adolescence, even among the non-clinical population. However, these adolescents were more likely to experience PTG than those who experienced NLEs after entering elementary school, indicating that the developmental stage at which NLEs occur may predict the level of PTG (8). Moreover, time elapsed after the trauma may be associated with negative outcomes. Such adolescents may go through positive psychological change from childhood to adolescence.

In the present study, path analysis demonstrated an association between perceived stress at the time of the NLE and PTG. Individuals with stable trait-resilience reported greater PTG, which reduced PTSD symptoms and current depressive syndrome. Model 1 demonstrated a mediating relationship between stress intensity at the time of NLE, PTG, and depression. Greater PTG was associated with lower levels of current depressive 


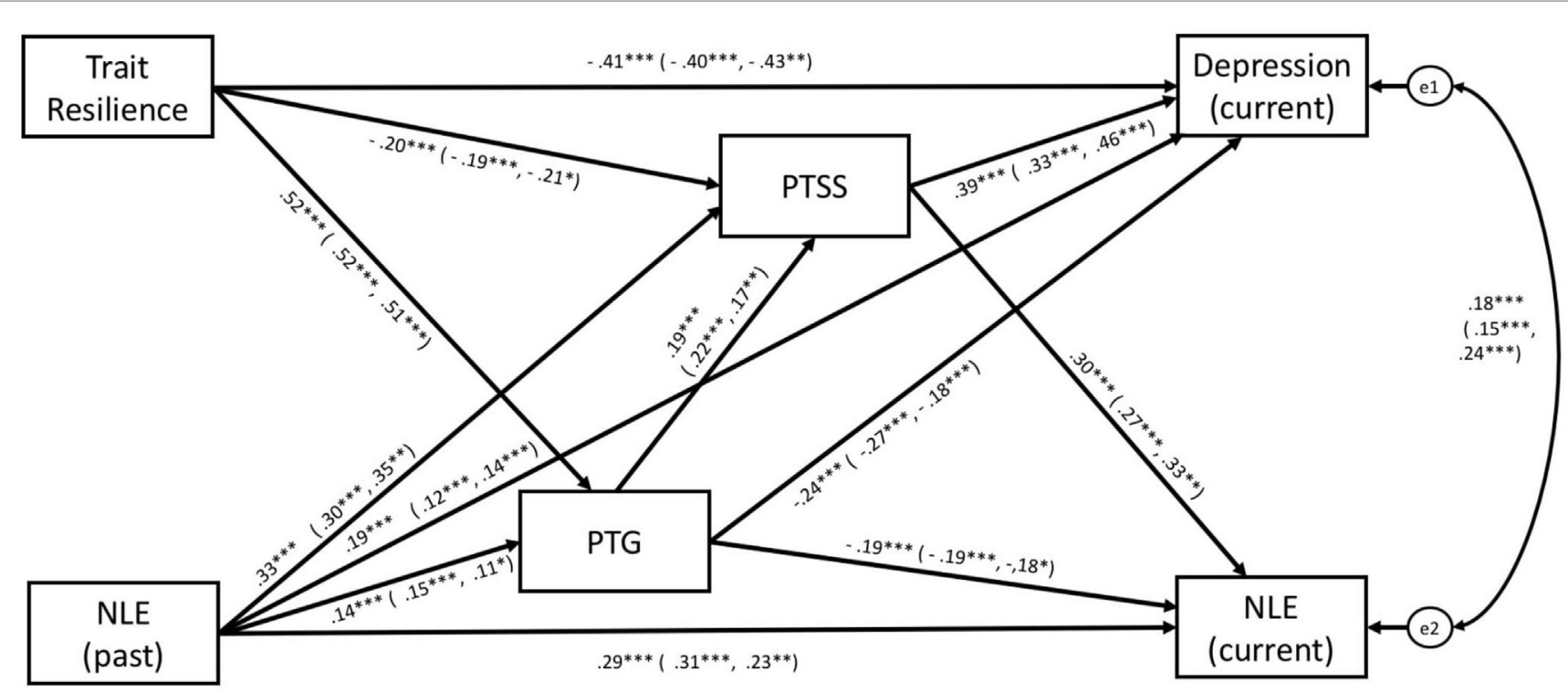

FIGURE 5 | Multi and mediated model (Model 4). Illustration of a mediation model in which trait-resilience and past stress are hypothesized to exert indirect effects on current negative outcomes (current depression and stress associated with NLEs) via PTSS and PTG. Note: standardized estimates of the direct and indirect effects on depressive symptoms are presented for all participants (male, female), respectively. Abbreviations: NLE, negative life event; PTSS, posttraumatic stress symptoms; PTG, posttraumatic growth.

symptoms. Model 2 was used to examine the mediating relationship between stress intensity at the time of NLE, PTSS, and depression. However, this model did not achieve critical GFI values. Model 3, which was constructed using multiple mediators (i.e., PTSS and PTG), indicated that NLEs directly influenced depression and PTSS, and that PTG mediated the association between NLEs and depression. Finally, Model 4 demonstrated the direct and indirect effects of perceived stress at the time of the NLE and trait-resilience, which were identified as predictors of current stress intensity (NLEs current) and depression. Our results indicated that these associations were mediated by PTSS and PTG.

Significant differences in some GFI values were observed across gender. For example, GFIs for Model 2 were acceptable among boys, while those for girls were slightly worse than the critical values. Although these findings suggest that gender plays a role in mediating these effects, the sample size may have influenced our results. Thus, further studies are required to clarify the effect of gender on these associations. The results of the present study are in accordance with those of a recent study, which indicated that adolescents who had been exposed to one or several traumatic experiences reported more internal and external problems than those who had internalizing and externalizing problems compared to those who had not been exposed to such experiences. The authors also demonstrated associations between cluster 2 and internal/external conflicts (49). Further studies are needed to examine whether current depressive symptoms might be a cause of (rather than an effect of) increased exposure to stressors and the degree of their negative affect.

The present study possesses some limitations of note. First, as our study utilized a cross-sectional design, we were unable to determine causal associations. The present study has struggled with the emerging consensus in the field regarding the issue of using cross-sectional data to test mediation (50). Second, there are several limitations to mention about the sample used in the present study. We were unable to account for the influence of socioeconomic status, as this information was not obtained in order to protect the privacy of the students. The unequal numbers of boys and girls is one of the major limitations. The present study was conducted at four general and three industrial high schools. The reason for the current gender distribution is that industrial high schools typically have a higher proportion of boys compared to girls. It is difficult to say that the youth of Fukui are representative of Japan unless more participants from other prefectures are included, and there has been, to date, no normative study of adolescent mental health problems conducted in Japan. Third, as the NLE questionnaire used in the present study included current NLEs, a group of students $(n=255)$ who reported recent NLEs were excluded in order to examine the effects of past NLEs on outcomes. Thus, we were unable to determine the full effects of past NLEs on outcomes for all participants. Based on Cook's definition of complex trauma (10), it might be possible to interpret the cluster 1 as "single incident trauma," cluster 2 as "complex trauma," and cluster 3 as "combined trauma." However, it is clear that these should be labeled with more scientific basis. Further studies are required to determine the effects of different types of trauma on mental health outcomes among non-clinical adolescents. Fourth, lack of the replicability of the cluster analysis (51) is another issue of the present study. The clusters failed to replicate across random subsamples even though similarities of patterns were observed across the groups (see Data Sheet S1 in Supplementary Material). It is necessary for the further studies 
to investigate by using alternative methods for providing internal validity. Finally, we did not examine the influence of other psychosocial factors such as social support (52), meaning-making (e.g., to improve adjustment to the stressful event) (53), and coping (54), and others (55) on PTG. Future studies should focus on such analyses in order to provide new directions for interventions following NLEs.

Despite these limitations, our results demonstrated an association between perceived stress associated with NLEs and current depressive symptoms, and that this association was mediated by PTSS and PTG in a large sample of Japanese adolescents. Moreover, our findings indicated that event intensity, NLE timing, and gender play a role in levels of emotional vulnerability during adolescence. Thus, clinicians and educators should consider the timing of NLE exposure (11) as well as the severity of NLEs in the promotion of social-cognitive skills (56) and trait-resilience.

\section{ETHICS STATEMENT}

The study protocol was approved by the ethical committee at the University of Fukui (approval number: 834). The purposes of the present study were announced at a meeting of high-school principals from Fukui Prefecture, at which time their assistance and cooperation were requested. Principals and individual classroom teachers provided consent to include 29 classes from seven high schools. A researcher and technician visited schools and conducted the study. The purposes and voluntary nature of the present study were explained to parents by a letter and to students orally and in writing, following which they were requested to submit a letter of consent if they wished to participate in the study. Participating students completed the questionnaires anonymously during regular class hours. Identifying information

\section{REFERENCES}

1. Grant KE, Compas BE, Thurm AE, McMahon SD, Gipson PY. Stressors and child and adolescent psychopathology: measurement issues and prospective effects. J Clin Child Adolesc Psychol (2004) 33:412-25. doi:10.1207/ s15374424jccp3302

2. Grant KE, Compas BE, Thurm AE, McMahon SD, Gipson PY, Campbell AJ, et al. Stressors and child and adolescent psychopathology: evidence of moderating and mediating effects. Clin Psychol Rev (2006) 26:257-83. doi:10.1016/j. cpr.2005.06.011

3. Deb S, Strodi E, Sun J. Academic-related stress among private secondary school students in India. Asian Educ Dev Stud (2014) 3:118-34. doi:10.1108/ AEDS-02-2013-0007

4. Weymouth BB, Buehler C, Zhou N, Henson RA. A meta-analysis of parent-adolescent conflict: disagreement, hostility, and youth maladjustment. J Fam Theory Rev (2016) 8:95-112. doi:10.1007/s10964-015-0348-3

5. Mann MJ, Kristjansson AL, Sigfusdottir ID, Smith ML. The impact of negative life events on young adolescents: comparing the relative vulnerability of middle level, high school, and college-age students. RMLE Online (2014) 38:1-13. doi:10.1080/19404476.2014.11462115

6. Stikkelbroek Y, Bodden DHM, Kleinjan M, Reijnders M, Van Baar AL. Adolescent depression and negative life events, the mediating role of cognitive emotion regulation. PLoS One (2016) 11:e0161062. doi:10.1371/journal. pone.0161062

7. Cuijpers P, Smit F, Unger F, Stikkelbroek Y, ten Have M, de Graaf R. The disease burden of childhood adversities in adults: a population-based study. Child Abuse Negl (2011) 35:937-45. doi:10.1016/j.chiabu.2011.06.005 included only gender, age, and class. Students took approximately 30 min to complete the questionnaires.

\section{AUTHOR CONTRIBUTIONS}

TF and MK were involved in recruiting the participants and conducting the experiment. SN and TF were involved in analyzing and interpreting data, and drafting the article. AT conceived of the study, participated in its design and coordination, and drafted the manuscript. All the authors have read and approved the final manuscript.

\section{FUNDING}

This work was supported by a Grant-in-Aid for "Creating a Safe and Secure Living Environment in the Changing Public and Private Spheres" from the Japan Science and Technology Corporation (JST)/Research Institute of Science and Technology for Society (RISTEX). The funders had no role in the study design, data collection and analysis, decision to publish, or preparation of the manuscript. This study was also partially supported by Scientific Research (B) (\#15H03106 to AT) and Challenging Exploratory Research (\#15K12720 to AT) from the Japan Society for the Promotion of Science, research grants from the Takeda Science Foundation (to AT) and from the Public Health Research Foundation (2013-2014) (TXF).

\section{SUPPLEMENTARY MATERIAL}

The Supplementary Material for this article can be found online at http://www.frontiersin.org/articles/10.3389/fpsyt.2018.00041/ full\#supplementary-material.

8. Arpawong TE, Rohrbach LA, Milam JE, Unger JB, Land H, Sun P, et al. Stressful life events and predictors of post-traumatic growth among high-risk early emerging adults. J Posit Psychol (2016) 11:1-14. doi:10.1080/17439760. 2014.994223

9. McLaughlin KA, Lambert HK. Child trauma exposure and psychopathology: mechanisms of risk and resilience. Curr Opin Psychol (2017) 14:29-34. doi:10.1016/j.copsyc.2016.10.004

10. Cook A, Spinazzola J, Ford J, Lanktree C, Blaustein M, Cloitre M, et al. Complex trauma in children and adolescents. Psychiatr Ann (2005) 35(5):390-8. doi:10.3928/00485713-20050501-05

11. Dunn EC, Nishimi K, Powers A, Bradley B. Is developmental timing of trauma exposure associated with depressive and post-traumatic stress disorder symptoms in adulthood? J Psychiatr Res (2017) 84:119-27. doi:10.1016/j. jpsychires.2016.09.004

12. Teicher MH, Samson JA. Childhood maltreatment and psychopathology: a case for ecophenotypic variants as clinically and neurobiologically distinct subtypes. Am JPsychiatry (2013) 170(10):1114-33. doi:10.1176/appi. ajp.2013.12070957

13. Buckholdt KE, Weiss NH, Young J, Gratz KL. Exposure to violence, posttraumatic stress symptoms, and borderline personality pathology among adolescents in residential psychiatric treatment: the influence of emotion dysregulation. Child Psychiatry Hum Dev (2015) 46:884-92. doi:10.1007/ s10578-014-0528-5

14. Grant KE, Compas BE, Stuhlmacher AF, Thurm AE, McMahon SD, Halpert JA. Stressors and child and adolescent psychopathology: moving from markers to mechanisms of risk. Psychol Bull (2003) 129:447-66. doi:10.1037/0033-2909.129.3.447 
15. Tedeschi RG, Calhoun LG. Posttraumatic growth: conceptual foundations and empirical evidence. Psychol Inq (2004) 15:1-18. doi:10.1207/ s15327965pli1501_01

16. Linley PA, Joseph S. Positive change following trauma and adversity: a review. J Trauma Stress (2004) 17:11-21. doi:10.1023/B:JOTS.0000014671.27856.7e

17. Shakespeare-Finch J, Lurie-Beck J. A meta-analytic clarification of the relationship between posttraumatic growth and symptoms of posttraumatic distress disorder. J Anxiety Disord (2014) 28:223-9. doi:10.1016/j.janxdis.2013.10.005

18. Galea S, Nandi A, Vlahov D. The epidemiology of post-traumatic stress disorder after disasters. Epidemiol Rev (2005) 27:78-91. doi:10.1093/epirev/mxi003

19. Calhoun LG, Tedeschi RG. The Foundations of posttraumatic growth: new considerations. Psychol Inq (2004) 15:93-102. doi:10.1207/s15327965pli1501_03

20. Tedeschi RG. Posttraumatic growth in combat veterans. J Clin Psychol Med Settings (2011) 18:137-44. doi:10.1007/s10880-011-9255-2

21. Ickovics JR, Meade CS, Kershaw TS, Milan S, Lewis JB, Ethier KA. Urban teens: trauma, posttraumatic growth, and emotional distress among female adolescents.J Consult Clin Psychol(2006)74:841-50.doi:10.1037/0022-006X.74.5.841

22. Milam JE, Ritt-Olson A, Unger JB. Posttraumatic growth among adolescents. J Adolesc Res (2004) 19:192-204. doi:10.1177/0743558403258273

23. Block JH, Block J. The role of ego-control and ego-resiliency in the organization of behavior. In: Collins WA, editor. Minnesota symposia on child psychology: Vol. 13. Development of cognition affect, and social relations. Hillsdale, NJ: Erlbaum. (1980). p. 39-101.

24. Farkas D, Orosz G. Ego-resiliency reloaded: a three-component model of general resiliency. PLoS One (2015) 10:e0120883. doi:10.1371/journal. pone. 0120883

25. Block J, Block JH. Venturing a 30-year longitudinal study. Am Psychol (2006) 61:315-27. doi:10.1037/0003-066X.61.4.315

26. Alessandri G, Eisenberg N, Vecchione M, Caprara GV, Milioni M. Egoresiliency development from late adolescence to emerging adulthood: a ten-year longitudinal study. JAdolesc (2016) 50:91-102. doi:10.1016/j. adolescence.2016.05.004

27. Taylor ZE, Sulik MJ, Eisenberg N, Spinrad TL, Silva KM, Lemery-Chalfant $\mathrm{K}$, et al. Development of ego-resiliency: relations to observed parenting and polymorphisms in the serotonin transporter gene during early childhood. Soc Dev (2014) 23:433-50. doi:10.1111/sode.12041

28. Duan W, Guo P, Gan P. Relationships among trait resilience, virtues, post-traumatic stress disorder, and posttraumatic growth. PLoS One (2015) 10:e0125707. doi:10.1371/journal.pone.0125707

29. Sarubin N, Wolf M, Giegling I, Hilbert S, Naumann F, Gutt D, et al. Neuroticism and extraversion as mediators between positive/negative life events and resilience. Pers Individ Dif (2015) 82:193-8. doi:10.1016/j.paid.2015.03.028

30. Peng L, Zhang J, Li M, Li P, Zhang Y, Zuo X, et al. Negative life events and mental health of Chinese medical students: the effect of resilience, personality and social support. Psychiatry Res (2012) 196:138-41. doi:10.1016/j. psychres.2011.12.006

31. Campbell-Sills L, Barlow DH, Brown TA, Hofmann SG. Effects of suppression and acceptance on emotional responses of individuals with anxiety and mood disorders. Behav Res Ther (2006) 44:1251-63. doi:10.1016/j.brat.2005. 10.001

32. Weiss DS, Marmar CR. The Impact of Event Scale: Revised. In: Wilson JP, Keane TM, editors. Assessing psychological trauma and PTSD. New York: Guilford Press (1997). p. 399-411.

33. Asukai N, Kato H, Kawamura N, Kim Y, Yamamoto K, Kishimoto J, et al. Reliability and validity of the Japanese-language version of the Impact of Event Scale-Revised (IES-R-J): four studies of different traumatic events. J Nerv Ment Dis (2002) 190:175-82. doi:10.1097/00005053-200203000-00006

34. Taku K, Calhoun LG, Tedeschi RG, Gil-Rivas V, Kilmer RP, Cann A. Examining posttraumatic growth among Japanese university students. Anxiety Stress Coping (2007) 20:353-67. doi:10.1080/10615800701295007

35. Tedeschi RG, Calhoun LG. The posttraumatic growth inventory: measuring the positive legacy of trauma. J Trauma Stress (1996) 9:455-71. doi:10.1007/ BF02103658

36. Birleson P, Hudson I, Buchanan DG, Wolff S. Clinical evaluation of a self-rating scale for depressive disorder in childhood (Depression Self-Rating Scale). J Child Psychol Psychiatry (1987) 28:43-60. doi:10.1111/j.1469-7610.1987. tb00651.x
37. Murata T. Examination of Birleson Depression Scale for school children in Japan. Jpn J Latest Psychiatry (1996) 1:131-8.

38. Block J, Kremen AM. IQ and ego-resiliency: conceptual and empirical connections and separateness. JPers Soc Psychol (1996) 70:349-61. doi:10.1037/0022-3514.70.2.349

39. Hata U, Onodera A. Development and validation of a Japanese version of the Ego-Resiliency Scale (ER89). (English). Jpn J Personal (2013) 22:37-47. doi:10.2132/personality.22.37

40. Baron RM, Kenny DA. The moderator-mediator variable distinction in social psychological research: conceptual, strategic, and statistical considerations. J Pers Soc Psychol (1986) 51:1173-82. doi:10.1037/0022-3514.51.6.1173

41. Holmes-Smith P. Introduction to structural equation modelling using LISREL. ACSPRI-Winter Training Program, Perth. ACSPRI-Winter Training Program. Perth (2017).

42. Bentler PM. Comparative fit indexes in structural models. Psychol Bull (1990) 107:238-46. doi:10.1037/0033-2909.107.2.238

43. Bollen KA. Structural Equations with Latent Variables. New York: Wiley (1989).

44. Browne MW, Cudeck R. Alternative ways of assessing model fit. Sociol Methods Res (1992) 21:230-58. doi:10.1177/0049124192021002005

45. Preacher KJ, Hayes AF. Asymptotic and resampling strategies for assessing and comparing indirect effects in multiple mediator models. Behav Res Methods (2008) 40(3):879-91. doi:10.3758/BRM.40.3.879

46. Nishikawa S, Sundbom E, Hägglöf B. Influence of perceived parental rearing on adolescent self-concept and internalizing and externalizing problems in Japan. J Child Fam Stud (2010) 19:57-66. doi:10.1007/s10826-009-9281-y

47. Steinberg LD. Adolescence. New York: McGraw-Hill Higher Education (2002).

48. Zhai Y, Liu K, Zhang L, Gao H, Chen Z, Du S, et al. The relationship between post-traumatic symptoms, parenting style, and resilience among adolescents in Liaoning, China: a cross-sectional study. PLoS One (2015) 10:e0141102. doi:10.1371/journal.pone.0141102

49. Yearwood K, Vliegen N, Chau C, Corveleyn J, Luyten P. Prevalence of exposure to complex trauma and community violence and their associations with internalizing and externalizing symptoms. JInterpers Violence (2017). doi: $10.1177 / 0886260517731788$

50. Maxwell SE, Cole DA. Bias in cross-sectional analyses of longitudinal mediation. Psychol Methods (2007) 12:23-44. doi:10.1037/1082-989X.12.1.23

51. Babbin SF, Velicer WF, Paiva AL, Brick LA, Redding CA. Replicating cluster subtypes for the prevention of adolescent smoking and alcohol use. Addict Behav (2015) 40:57-65. doi:10.1016/j.addbeh.2014.09.001

52. Jia X, Ying L, Zhou X, Wu X, Lin C. The effects of extraversion, social support on the posttraumatic stress disorder and posttraumatic growth of adolescent survivors of the Wenchuan earthquake. PLoS One (2015) 10:e0121480. doi:10.1371/journal.pone.0121480

53. Park CL. Making sense of the meaning literature: an integrative review of meaning making and its effects on adjustment to stressful life events. Psychol Bull (2010) 136:257-301. doi:10.1037/a0018301

54. Pfefferbaum B, Nitiéma P, Jacobs AK, Noffsinger MA, Wind LH, Allen SF. Review of coping in children exposed to mass trauma: measurement tools, coping styles, and clinical implications. Prehosp Disaster Med (2016) 31(2):169-80. doi:10.1017/S1049023X16000169

55. Fujisawa TX, Jung M, Kojima M, Saito DN, Kosaka H, Tomoda A. Neural basis of psychological growth following adverse experiences: a resting-state functional MRI study. PLoS One (2015) 10:e0136427. doi:10.1371/journal. pone. 0136427

56. Sharp C, Fonagy P, Allen JG. Posttraumatic stress disorder: a social-cognitive perspective. Clin Psychol Sci Pract (2012) 19:229-40. doi:10.1111/cpsp.12002

Conflict of Interest Statement: The authors declare that the research was conducted in the absence of any commercial or financial relationships that could be construed as a potential conflict of interest.

Copyright $\odot 2018$ Nishikawa, Fujisawa, Kojima and Tomoda. This is an open-access article distributed under the terms of the Creative Commons Attribution License (CC $B Y)$. The use, distribution or reproduction in other forums is permitted, provided the original author(s) and the copyright owner are credited and that the original publication in this journal is cited, in accordance with accepted academic practice. No use, distribution or reproduction is permitted which does not comply with these terms. 